\section{Kidney \\ Blood Pressure Research}

\title{
The Calcium-Sensing Receptor Gene Polymorphism rs1801725 and Calcium- Related Phenotypes in Hemodialysis Patients
}

\author{
Alicja E. Grzegorzewskaa ${ }^{a}$ Dariusz Bednarskib ${ }^{\text {Monika Świderska }}{ }^{a}$ \\ Adrianna Mostowskac Paweł P. Jagodzińskic \\ aDepartment of Nephrology, Transplantology and Internal Diseases, Poznan University of Medical \\ Sciences, Poznań, bStudent Nephrology Research Group, Department of Nephrology, Transplantology \\ and Internal Diseases, Poznan University of Medical Sciences, Poznań, 'Department of Biochemistry and \\ Molecular Biology, Poznan University of Medical Sciences, Poznań, Poland
}

\section{Key Words}

Calcium • Calcium-sensing receptor gene - Cinacalcet • Hemodialysis • Nephrolithiasis • Parathyroid hormone $\cdot$ Phosphorus • Survival $\bullet$ Vitamin D pathway genes

\begin{abstract}
Background/Aims: The calcium-sensing receptor gene (CASR) rs1801725 variant is responsible for a non-conservative amino-acid change (A986S) in the calcium-sensing receptor cytoplasmic tail. We hypothesized that rs1801725 polymorphism might be helpful in understanding Ca-related abnormalities in HD patients. Methods: In 1215 subjects (245 on cinacalcet), we determined the associations of rs 1801725 with secondary hyperparathyroidism (sHPT)related laboratory parameters, PTH-decreasing effect of cinacalcet hydrochloride, coronary artery disease (CAD), myocardial infarction (MI), nephrolithiasis-related ESRD, and mortality. CASR rs7652589(A<G)_rs1801725(G>T) haplotypes and rs1801725 epistatic interactions with vitamin $D$ signaling pathway genes were examined for associations with selected phenotypes. Results: The rs 1801725 variant allele showed an increasing independent effect on plasma PTH $\left(P_{\text {corrected }}=0.009\right)$. CASR rs7652589_rs1801725 AT haplotype was associated with 1.7-fold higher frequency of PTH levels over $437 \mathrm{pg} / \mathrm{mL}$ than the reference haplotype $\mathrm{GG}(P=0.001)$. CASR rs7652589_rs1801725 AG haplotype was 1.5-fold more frequent in nephrolithiasis-related ESRD than the $G G$ haplotype $(P=0.004)$. There were no significant associations between rs1801725, CAD, MI, and response to cinacalcet. Variant homozygosity of rs 1801725 correlated independently with higher infection-related mortality compared with heterozygosity (HR 7.95,
\end{abstract}




\section{Kidney Blood Pressure Research}

Kidney Blood Press Res 2018;43:719-734

\begin{tabular}{l|l}
\hline DOI: $10.1159 / 000489747$ & (C) 2018 The Author(s). Published by S. Karger AG, Basel
\end{tabular}

Published online: 16 May, 2018

www.karger.com/kbr

95\%CI $2.15-29.37, \mathrm{P}=0.003)$ and major homozygosity (HR 5.89, 95\%CI $1.69-20.55, \mathrm{P}=$ $0.040)$. CASR rs 1801725 did not show epistatic interactions with vitamin $D$ signaling pathway genes concerning tested associations. Conclusion: The variant allele of CASR rs 1801725 solely and together with the variant allele of rs7652589 increases risk of more advanced sHPT. Homozygosity of the rs 1801725 variant allele contributes to infection-related mortality in HD patients.

(C) 2018 The Author(s)

Published by S. Karger AG, Basel

\section{Introduction}

The majority of end-stage renal disease (ESRD) patients demonstrates secondary hyperparathyroidism (sHPT), which enhances with prolongation of renal replacement therapy (RRT) duration [1] and requires parathyroidectomy (PTx) in the most severe cases [2]. Mineral bone disorders, including SHPT, are closely associated with cardiovascular complications and contribute to morbidity and mortality in hemodialysis (HD) subjects [3]. Serum parathyroid hormone (PTH) concentrations and other features of sHPT [4-7], as well as treatment with cinacalcet [7-9], seem to be associated with calcium-sensing receptor gene (CASR) single nucleotide polymorphisms (SNPs).

In our study, the CASR rs1801725 SNP was selected for genotyping. The rs1801725 SNP encodes a missense variant in the $C A S R$ exon 7, which leads to a non-conservative amino-acid change (A986S) in the calcium-sensing receptor (CaSR) cytoplasmic tail. CaSR is sensitive to ionized Ca concentration and regulates PTH secretion in parathyroid cells [10]. In 1999, the variant allele of rs1801725 was associated with higher circulating calcium levels in healthy women [11]. Higher calcium concentrations in subjects harboring the variant allele of rs1801725 were reported as associated with decreased sensitivity of the CaSR to calcium due to the production of a less active receptor by the variant allele of rs1801725 [10, 12]. However, the rs1801725 polymorphism was not a predictor of serum calcium levels either in primary HPT [13] or sHPT [7]. Moreover, two functional studies documented the normal activity of CaSR coded by the variant allele of rs1801725 SNP $[14,15]$. Therefore, the impact of the variant rs1801725 allele on HPT phenotypes cannot be simply explained by a less active CaSR, and is still open for investigations.

GWAS concerning serum PTH concentrations in European ancestry showed that among genes showing the most influential association with serum PTH levels are rs6127099 upstream of CYP24A1 that encodes the catabolic enzyme for 1,25-dihydroxyvitamin D and 25-dihydroxyvitamin D, and rs73186030 near CASR [16]. Other CASR SNPs, rs7652589 [6] and rs1801725 [7], were individually involved in serum PTH levels in HD patients. It is not clear whether haplotypes of these SNPs are also associated with PTH levels, and whether they interact with vitamin $D$ pathway genes or not.

The CASR rs1801725 polymorphism was not associated with the risk of nephrolithiasis development in a Chinese population [17]. In HD patients, rs7652589 SNP correlated with nephrolithiasis-related ESRD without interaction with SNPs of vitamin D signaling pathway genes [6].

The significant associations of CASR rs1801725 with calcium-related outcomes (coronary heart disease - CAD, myocardial infarction - MI, hypertension, stroke, osteoarthritis, osteoporosis, and nephrolithiasis) were not found in Genome-Wide Meta-Analysis [18]. However, in angiographically defined CAD patients, the variant allele of CASR rs1801725 was reported to be an independent predictor of CAD, MI, all-cause mortality, and cardiovascular mortality [19].

Data mentioned above indicate that CASR rs1801725 affecting serum $\mathrm{Ca}, \mathrm{Mg}, \mathrm{P}$, and PTH, might be involved in Ca-related phenotypes in HD patients solely, with other CASR SNPs, or interacting with vitamin D signaling pathway genes.

Calcimimetics, allosteric activators of CaSR, provide an effective means of reducing PTH secretion in HD patients [20]. Studies of the association between CASR and the response 


\section{Kidney Blood Pressure Research}

to treatment with the calcimimetic agent cinacalcet included several CASR SNPs [6-9] and showed that CASR R990G (rs1042636) influences the response to calcimimetics in patients with sHPT [9].

Our study aimed to determine the associations of the CASR rs1801725 SNP with sHPT-related laboratory parameters, PTH-decreasing effect of treatment with cinacalcet hydrochloride, Ca-related morbidities (CAD, MI, nephrolithiasis-related ESRD) as well as mortality of HD patients. CASR rs7652589_rs1801725 haplotypes and CASR rs1801725 SNP epistatic interactions with vitamin D signaling pathway genes were also examined for associations with serum PTH levels, PTH-lowering response to cinacalcet, and nephrolithiasis-related ESRD.

\section{Materials and Methods}

\section{Patients}

ESRD patients whose current method of RRT was intermittent HD were enrolled in the study. HD subjects who had undergone renal transplantation (RTx) but returned to HD treatment after graft loss were excluded as living with renal graft influences parameters related to sHPT $[21,22]$ and prolongs lifetime on RRT [23].

Patient enrollment began in January 2009 and ended in October 2017. Among the studied group (n = 1215), there was 1017 (83.7\%) individuals genotyped for CASR rs7652589 SNP and vitamin D signaling pathway gene SNPs in our previous study [6].

The clinical management of patients was decided by an individual physician and not affected by the study. The dietary and pharmacological treatment of all patients was based on a standard of care according to the physician. Guidelines for this treatment based on international recommendations [24,25]. Nephrologists had been prescribing calcium carbonate or calcium acetate due to hyperphosphataemia without hypercalcemia or due to hypocalcemia. Sevelamer hydrochloride was used in hypercalcaemic/hyperphosphatemic patients. Among vitamin D supplements, alfacalcidol (Alfadiol, GlaxoSmithKline Pharmaceuticals SA, Poland) was the most frequently used. None of the patients was administered intravenous vitamin $\mathrm{D}$ analogs.

Patients with serum PTH levels equal to or exceeding $500 \mathrm{pg} / \mathrm{mL}$ could receive treatment with cinacalcet hydrochloride (reimbursed by the National Health Fund - NHF). Treatment with cinacalcet (Mimpara, Amgen Europe, Breda, Netherlands) at a daily dose of $30 \mathrm{mg}$ was started in 245 patients with PTH exceeding $500 \mathrm{pg} / \mathrm{mL}$. Among this group, there were 161 subjects analyzed in the previous study concerning CASR rs7652589 SNP [6]. Patients who showed serum PTH of $1000 \mathrm{pg} / \mathrm{mL}$ or higher before the planned treatment with cinacalcet underwent 99mTc-MIBI scintigraphy. Only those without scintigraphic suspicion of parathyroid gland adenoma qualified for this treatment; the remaining underwent PTx if possible (no clinical contraindications, written informed consent obtained). In the cinacalcet group, serum PTH was checked every four weeks, and cinacalcet doses were increased up to $180 \mathrm{mg}$ per day, if well tolerated, until the PTH concentration decreased at least by $40 \%$ of the initial level, which was considered as a satisfactory response to treatment. Patients who did not exhibit sufficiently reduced serum PTH were referred to as non-responders to cinacalcet.

Blood samples for Ca, P, ALP, and PTH were routinely taken from all HD patients at the onset of RRT. Periodical measurements were conducted every 1-3 months for Ca and P and every 6-12 months for ALP and PTH. Differences in the frequency of measurements were dependent on the intrinsic regulations of the respective dialysis centers.

All study subjects were Caucasians of Polish origin.

\section{Patient Data}

Demographic and clinical parameters including date of birth, gender, chronic kidney disease causing ESRD and requiring RRT, time of the start of RRT, treatment with peritoneal dialysis before HD initiation, presence of CAD, a history of MI, and PTx were recorded by a review of the medical charts and by personal communications with the nephrologists responsible for the treatment of patients in the dialysis centers. The 


\section{Kidney Blood Pressure Research}

patient data concerning CAD, MI, and PTx were re-evaluated every year from the initiation to the end of the enrollment period.

Laboratory parameters included serum concentrations of total Ca, P, and intact PTH, as well as serum activity of total ALP; all serum concentrations were determined using routine laboratory methods. The laboratory data were evaluated in our database every year: the last four results obtained while the patient was in stable condition were collected as long as their number was not limited by a short HD vintage. For analysis, each laboratory parameter was represented as a mean value of up to 4 results obtained during the last period of HD duration. In patients who underwent PTx or were treated with cinacalcet, laboratory data obtained before those treatments were included in the analysis. Analyses of the CASR association with serum calcium were also performed using the lowest Ca levels among up to 4 last collected values, if patients were receiving Ca-containing drugs. The CASR associations with circulating phosphorus were also explored using the highest $\mathrm{P}$ levels among up to 4 previous collected values if patients were taking phosphate-binding drugs.

Additionally, plasma 25(OH)D levels were determined in 245 blindly selected HD patients. Blood samples for 25(OH)D were taken in the winter to avoid differences in sunlight exposure between patients who sunbathed and those who did not. Plasma 25(OH)D concentrations were measured in HD patients who had not been treated with vitamin D or had stopped vitamin D treatment at least three weeks earlier.

In patients treated with cinacalcet, serum PTH concentrations preceding the initiation of cinacalcet administration and those measured 1-2 months after implementation of the maximum cinacalcet dose were collected. However, serum PTH concentrations were obtained no longer than 12 months from the beginning of treatment.

For the survival analysis, the dates of death and kidney transplantation were recorded. The causes of death were categorized as cardiac (reported as myocardial infarction, sudden cardiac death, severe arrhythmias, cardiomyopathies, or cardiac failure), vascular (reported as cerebrovascular events, cerebral stroke, or generalized atherosclerosis), cancer-related, infection-related, or other. The last evaluation of patient outcomes was performed in January 2018.

\section{Genotyping}

Genotyping was performed using leukocyte DNA obtained from the blood.

The CASR rs1801725 SNP was genotyped in enrolled HD patients. Analysis of CASR rs1801725 variant was performed using pre-designed TaqMan SNP Genotyping Assay according to the manufacturer's instructions provided by Applied Biosystems (Applied Biosystems, Foster City, CA).

CASR rs7652589 SNP and vitamin D signaling pathway gene SNPs were genotyped using previously described methods $[6,26]$ in all HD patients who were not tested for our earlier studies. Genetic testing included the SNPs in the following vitamin D signaling pathway genes: group-specific component protein, $G C$ (rs7041, rs1155563, rs2298849), retinoid X receptor alpha, RXRA (rs10881578, rs10776909, rs749759), and vitamin D receptor, VDR (rs1544410, rs2228570).

Quality control was ensured by running $10 \%$ of the samples in duplicate. Only samples providing unequivocal results were taken for analysis.

Distributions of tested polymorphisms were in concordance with the Hardy-Weinberg equilibrium (HWE).

\section{Statistical analysis}

The results are presented as percentages for categorical variables or as the median and range (minimum-maximum) for continuous variables because the majority of continuous variables were nonnormally distributed as tested by the Shapiro-Wilk test.

Demographic, clinical and laboratory data were compared in HD subjects stratified by CASR rs1801725 genotypes. The results were analyzed using inheritance models (dominant, recessive, and additive). Continuous variables were compared using the Mann-Whitney test. For comparison of categorical variables, Chi-square test or Fisher`s test was used, as appropriate. A receiver operating characteristic (ROC) curve analysis was applied to create serum PTH cut-off value for PTH as a dichotomous variable.

Departure from HWE was determined by Chi-squared analysis ( $P>0.05$ for equilibrium). Polymorphisms were tested for trends in association using the Chi-square test if two groups were compared 


\section{Kidney Blood Pressure Research}

Kidney Blood Press Res 2018;43:719-734

\begin{tabular}{l|l}
\hline DOI: $10.1159 / 000489747$ & (C) 2018 The Author(s). Published by S. Karger AG, Basel
\end{tabular}

Published online: 16 May, 2018

www.karger.com/kb

Grzegorzewska et al.: Calcium-Sensing Receptor Gene in HD Patients

or the Cochran-Armitage test if three groups were compared $\left(\mathrm{P}_{\text {trend }}\right)$. Genotype distributions were compared between the tested groups using the standard Chi-square test $\left(\mathrm{P}_{\text {genotype }}\right)$.

For categorical variables, Odds ratio (OR) and 95\% confidence intervals (CIs) for OR were calculated to quantify how strongly the presence or absence of tested allele, genotype or haplotype is associated with the presence or absence of selected phenotypes of HD patients. Chi-square test or Fisher's test was used for statistical evaluation of OR. All probabilities were two-tailed.

To determine the association of CASR SNP with serum PTH level among other relevant patient characteristics, we used the primary logistic regression models with subsequent stepwise logistic regression and backward elimination for selection of significant variables among other possible determinants of serum PTH.

Survival probability since the onset of RRT was analyzed concerning CASR genotypes. This analysis was also performed using dominant, recessive and additive models of inheritance. The Kaplan-Meier method with the log-rank test was used to estimate significant differences in the cumulative proportion of survival curves characterizing the genotype groups in each model of inheritance. The Cox proportional hazard model was applied to show whether and to which extend the effect of a unit increase in a covariate was multiplicative concerning the hazard rate of death. Cox proportional hazard model was also applied in multivariate analyses assessing the contribution of demographics and clinical measures to mortality.

The previously mentioned statistical analyses were performed using Statistica version 12 (Stat Soft, Inc., Tulsa, OK, USA) and R software, version 3.4.0.

Haplotype frequencies were estimated using the Haploview 4.2 software (http://www.broad.mit.edu/ $\mathrm{mpg} /$ haploview/). Haplotypes were statistically analyzed if their incidence in the examined group was over 1\%. Epistatic interactions between CASR SNP and vitamin D signaling pathway gene SNPs were analyzed using multifactor dimensionality reduction (MDR) method [27].

Values of $\mathrm{P}<0.05$ were considered significant in the epistatic interaction, multiple regression, and survival probability analyses. Bonferroni corrected $P$ values were deemed to be significant in statistical analyzes involving OR $(\% \% \mathrm{CI})$ calculations.

\section{Ethical approval}

The research design was approved by the Institutional Review Board of Poznan University of Medical Sciences, Poland. Informed consent was obtained from all study participants. The study was carried out following the approved guidelines.

\section{Results}

\section{Patient characteristics}

The analysis of demographic, clinical and laboratory data of HD patients, stratified by genotypes of $C A S R$ rs1801725, revealed no significant differences in the examined data, including the prevalence of nephrolithiasis-related ESRD, CAD, and MI (Table 1).

\section{An association of CASR rs1801725 SNP with laboratory parameters related to SHPT}

Table 2 shows laboratory parameters related to SHPT in HD subjects classified by the CASR rs1801725 genotypes. Patients harboring the variant allele of rs1801725 revealed higher PTH levels than homozygotes of the major allele (the dominant inheritance model). According to a ROC curve analysis, a PTH concentration differing patients bearing the variant allele from those being homozygotes of the major allele was shown to be $437 \mathrm{pg} / \mathrm{mL}$ (AUC 0.554 , SE 0.018, P = 0.002). Prevalence of PTH results over $437 \mathrm{pg} / \mathrm{mL}$ was about 1.5 -fold higher in subjects bearing the variant allele of rs1801725 (Table 3).

Total calcium and phosphorus concentrations, as well as total alkaline phosphatase (ALP) activity, did not present significant differences in models of inheritance (Table 2). When the lowest calcium concentrations and the highest phosphorus levels among the respective last four collected values were taken into account in the analysis, we also did not show significant associations with CASR rs1801725 SNP (Table 4). 


\section{Kidney Blood Pressure Research}

Table 1. Differences in selected data of hemodialysis patients concerning CASR rs1801725 polymorphic variants. ${ }^{\mathrm{a}}$ Mann Whitney test for quantitative variables or chi-squared test for categorical variables, ${ }^{\mathrm{b}}$ Fisher's test, ${ }^{c}$ Not significant after a Bonferroni correction $(\mathrm{P}>0.004)$. Results are presented as median and range (minimum-maximum) or the number of patients presenting indicated parameter with the $\%$ of the total of patients tested in each genotype group shown in parentheses. Conversion factors to SI units are as follows: for $25(\mathrm{OH}) \mathrm{D}-1 \mathrm{ng} / \mathrm{mL}=2.496 \mathrm{nmol} / \mathrm{L}$. Abbreviations: BMI - body mass index, CASR - calcium-sensing receptor gene, ESRD - end-stage renal disease, PD - peritoneal dialysis, RRT - renal replacement therapy

\begin{tabular}{|c|c|c|c|c|c|c|}
\hline Parameter & $\begin{array}{c}\mathrm{GG} \\
\mathrm{n}=840\end{array}$ & $\begin{array}{c}\text { GT } \\
n=347\end{array}$ & $\begin{array}{c}\text { TT } \\
n=28\end{array}$ & $\begin{array}{c}\text { Mode of } \\
\text { inheritance }\end{array}$ & Odds ratio $(95 \% \mathrm{CI})$ & $P$ value ${ }^{a}$ \\
\hline \multirow{3}{*}{ Male gender, $\mathrm{n}=674$} & \multirow{3}{*}{$458(54.5)$} & \multirow{3}{*}{$200(57.6)$} & \multirow{3}{*}{$16(57.1)$} & TT+ GT vs GG & $1.133(0.886-1.449)$ & 0.319 \\
\hline & & & & $\mathrm{TT}$ vs $\mathrm{GT}+\mathrm{GG}$ & $1.072(0.503-2.286)$ & 0.857 \\
\hline & & & & TT vs GG & $1.112(0.520-2.380)$ & 0.784 \\
\hline \multirow{3}{*}{$\begin{array}{l}\text { Age at RRT onset, years } \\
\mathrm{n}=1215\end{array}$} & \multirow{3}{*}{$\begin{array}{c}62.4 \\
(11.8-91.7)\end{array}$} & \multirow{3}{*}{$\begin{array}{c}60.7 \\
(11.1-89.8)\end{array}$} & \multirow{3}{*}{$\begin{array}{c}58.9 \\
(26.3-84.6)\end{array}$} & $\mathrm{TT}+\mathrm{GT}$ vs GG & & $0.042^{\mathrm{c}}$ \\
\hline & & & & TT vs. GT+GG & & 0.491 \\
\hline & & & & TT vs. GG & & 0.367 \\
\hline \multirow{3}{*}{$\begin{array}{l}\text { Diabetic nephropathy, } \\
\mathrm{n}=371\end{array}$} & \multirow{3}{*}{$250(29.8)$} & \multirow{3}{*}{$113(32.6)$} & \multirow{3}{*}{$8(28.6)$} & $\mathrm{TT}+\mathrm{GT}$ vs GG & $1.124(0.865-1.461)$ & 0.381 \\
\hline & & & & TT vs GT+GG & $0.908(0.396-2.081)$ & 0.820 \\
\hline & & & & TT vs GG & $0.944(0.410-2.172)$ & 0.892 \\
\hline \multirow{3}{*}{$\begin{array}{l}\text { Chronic } \\
\text { glomerulonephritis, } \\
\mathrm{n}=149\end{array}$} & \multirow{3}{*}{$94(11.2)$} & \multirow{3}{*}{$53(15.3)$} & \multirow{3}{*}{$2(7.1)$} & $\mathrm{TT}+\mathrm{GT}$ vs GG & $1.364(0.954-1.950)$ & 0.088 \\
\hline & & & & TT vs $\mathrm{GT}+\mathrm{GG}$ & $0.544(0.128-2.317)$ & $0.565^{b}$ \\
\hline & & & & TT vs GG & $0.610(0.143-2.613)$ & $0.760^{\mathrm{b}}$ \\
\hline \multirow{3}{*}{$\begin{array}{l}\text { Hypertensive } \\
\text { nephropathy, } \mathrm{n}=255\end{array}$} & \multirow{3}{*}{$183(21.8)$} & \multirow{3}{*}{$66(19.0)$} & \multirow{3}{*}{$6(21.4)$} & $\mathrm{TT}+\mathrm{GT}$ vs GG & $0.853(0.629-1.157)$ & 0.307 \\
\hline & & & & $\mathrm{TT}$ vs $\mathrm{GT}+\mathrm{GG}$ & $1.027(0.412-2.561)$ & 0.954 \\
\hline & & & & TT vs GG & $0.979(0.391-2.451)$ & 0.964 \\
\hline \multirow{3}{*}{$\begin{array}{l}\text { Nephrolithiasis-related } \\
\text { ESRD, } n=99\end{array}$} & \multirow{3}{*}{$72(8.6)$} & \multirow{3}{*}{$25(7.2)$} & \multirow{3}{*}{$2(7.1)$} & $\mathrm{TT}+\mathrm{GT}$ vs GG & $0.828(0.522-1.311)$ & 0.420 \\
\hline & & & & $\mathrm{TT}$ vs $\mathrm{GT}+\mathrm{GG}$ & $0.864(0.202-3.697)$ & $1.000^{\mathrm{b}}$ \\
\hline & & & & TT vs GG & $0.821(0.191-3.527)$ & $1.000^{\mathrm{b}}$ \\
\hline \multirow{3}{*}{$\begin{array}{l}\text { RRT duration, years, } \\
\mathrm{n}=1215\end{array}$} & 5.33 & 5.13 & 4.03 & TT+ GT vs GG & & 0.716 \\
\hline & $(0.008-28.30)$ & $(0.21-26.52)$ & $(0.009-14.49)$ & TT vs GT+GG & & $0.045^{c}$ \\
\hline & & & & $\begin{array}{c}\text { TT vs GG } \\
\text { TT+ GT vs GG }\end{array}$ & $1.877(0.916-3.850)$ & $\begin{array}{l}0.045^{c} \\
0.081\end{array}$ \\
\hline $\begin{array}{l}\text { PD as the first method of } \\
\text { RRT } n=31\end{array}$ & $17(2.0)$ & $13(3.7)$ & $1(3.6)$ & TT vs GT+GG & $1.428(0.188-10.860)$ & $0.519 \mathrm{~b}$ \\
\hline & & & & TT vs GG & $1.793(0.230-13.970)$ & $0.449^{\mathrm{b}}$ \\
\hline BMI $\mathrm{kg} / \mathrm{m}^{2}$ & 25.6 & 25.4 & 24.5 & $\mathrm{TT}+\mathrm{GT}$ vs GG & & 0.386 \\
\hline$n=1215$ & $(128-592)$ & $(147-44.2)$ & $\begin{array}{c}24.5 \\
(19.6-36.0)\end{array}$ & $\mathrm{TT}$ vs GT+GG & & 0.970 \\
\hline & & & & TT vs GG & & 0.956 \\
\hline & & & & $\mathrm{TT}+\mathrm{GT}$ vs GG & $0.898(0.699-1.152)$ & 0.396 \\
\hline $\begin{array}{l}\text { Patients with coronary } \\
\text { arty }\end{array}$ & $342(40.7)$ & $133(38.3)$ & $10(35.7)$ & TT vs GT+GG & $0.833(0.381-1.820)$ & 0.646 \\
\hline & & & & TT vs GG & $0.809(0.369-1.774)$ & 0.596 \\
\hline Patients with a history & & & & TT+ GT vs GG & $1.051(0.784-1.409)$ & 0.740 \\
\hline of myocardial infarction, & $181(21.5)$ & $77(22.2)$ & $7(25.0)$ & TT vs GT+GG & $1.200(0.505-2.855)$ & 0.679 \\
\hline $\mathrm{n}=265$ & & & & TT vs GG & $1.214(0.508-2.900)$ & 0.663 \\
\hline & & & & $\mathrm{TT}+\mathrm{GT}$ vs GG & $1.600(0.757-3.385)$ & 0.215 \\
\hline $\begin{array}{l}\text { Parathyroidectomized } \\
\text { patients, } n=29\end{array}$ & $17(2.0)$ & $12(3.5)$ & $0(0)$ & TT vs GT+GG & $0.689(0.041-11.560)$ & $1.000^{\mathrm{b}}$ \\
\hline & & & & TT vs GG & $0.826(0.048-14.070)$ & $1.000^{\mathrm{b}}$ \\
\hline & & & & $\mathrm{TT}+\mathrm{GT}$ vs GG & $1.304(0.970-1.753)$ & 0.078 \\
\hline Patients treated with & $158(18.8)$ & $82(23.6)$ & $5(17.9)$ & $\mathrm{TT}$ vs $\mathrm{GT}+\mathrm{GG}$ & $0.858(0.323-2.280)$ & 0.758 \\
\hline & & & & TT vs GG & $0.938(0.351-2.506)$ & 0.899 \\
\hline 25-hydroxyvitamin D, & 13.8 & 12.8 & 25.7 & $\mathrm{TT}+\mathrm{GT}$ vs GG & & 0.603 \\
\hline $\mathrm{ng} / \mathrm{mL}$ & $(3-42)$ & $(7-23.4)$ & $(13.6-40)$ & $\mathrm{TT}$ vs $\mathrm{GT}+\mathrm{GG}$ & & 0.066 \\
\hline $\mathrm{n}=245$ & $\mathrm{n}=174$ & $\mathrm{n}=67$ & $\mathrm{n}=4$ & TT vs GG & & 0.079 \\
\hline
\end{tabular}

Table 2. Associations of CASR rs1801725 polymorphic variants with plasma concentrations of calcium, phosphorus, and PTH in hemodialysis patients $(\mathrm{n}=1215)$, ${ }^{\text {a }}$ Mann Whitney test. Results are presented as median and range (minimum-maximum). $\mathrm{P}$ values significant after a Bonferroni correction $(\mathrm{P}<0.004)$ are indicated using bold font. Conversion factors to SI units are as follows: for alkaline phosphatase $-1 \mathrm{U} / \mathrm{L}=$ $0.0167 \mu \mathrm{kat} / \mathrm{L}$, for calcium - $1 \mathrm{mg} / \mathrm{dL}=0.25 \mathrm{mmol} / \mathrm{L}$, for phosphorus $-1 \mathrm{mg} / \mathrm{dL}=0.323 \mathrm{mmol} / \mathrm{L}$, for PTH $1 \mathrm{pg} / \mathrm{mL}=0.1061 \mathrm{pmol} / \mathrm{L}$. Abbreviations: ALP - alkaline phosphatase, CASR - calcium sensing receptor gene, PTH - parathyroid hormone

\begin{tabular}{|c|c|c|c|c|c|}
\hline Parameter & $\begin{array}{c}\mathrm{GG} \\
\mathrm{n}=840\end{array}$ & $\begin{array}{c}\text { GT } \\
n=347\end{array}$ & $\begin{array}{c}\text { TT } \\
n=28\end{array}$ & Mode of inheritance & $P$ value ${ }^{a}$ \\
\hline Total calcium, mg/dL & $\begin{array}{c}8.83 \\
(5.4-12.80)\end{array}$ & $\begin{array}{c}8.94 \\
(6.65-11.41)\end{array}$ & $\begin{array}{c}8.86 \\
(7.04-11.70)\end{array}$ & $\begin{array}{c}\text { TT+ GT vs GG } \\
\text { TT vs. GT+GG } \\
\text { TT vs GG }\end{array}$ & $\begin{array}{l}0.161^{\mathrm{a}} \\
0.970^{\mathrm{a}} \\
0.918^{\mathrm{a}}\end{array}$ \\
\hline Phosphorus, mg/dL & $\begin{array}{c}4.96 \\
(1.95-11.27)\end{array}$ & $\begin{array}{c}5.10 \\
(1.75-10.99)\end{array}$ & $\begin{array}{c}4.88 \\
(3.44-7.28)\end{array}$ & $\begin{array}{c}\text { TT+ GT vs GG } \\
\text { TT vs. GT+GG } \\
\text { TT vs GG }\end{array}$ & $\begin{array}{l}0.135^{\mathrm{a}} \\
0.695^{\mathrm{a}} \\
0.596^{\mathrm{a}}\end{array}$ \\
\hline PTH, pg/mL & $\begin{array}{c}347.5 \\
(7.3-3757)\end{array}$ & $\begin{array}{c}415 \\
(12.9-3269.7)\end{array}$ & $\begin{array}{c}465.3 \\
(80.1-1639.5)\end{array}$ & $\begin{array}{c}\mathrm{TT}+\mathrm{GT} \text { vs GG } \\
\mathrm{TT} \text { vs } \mathrm{GT}+\mathrm{GG} \\
\text { TT vs GG }\end{array}$ & $\begin{array}{l}0.002 \\
0.476 \\
0.311\end{array}$ \\
\hline Total ALP, U/L & $\begin{array}{c}97.8 \\
(15-1684)\end{array}$ & $\begin{array}{c}97 \\
(13.5-977.3)\end{array}$ & $\begin{array}{c}87 \\
(44-425.3)\end{array}$ & $\begin{array}{c}\text { TT+ GT vs GG } \\
\text { TT vs GT+GG } \\
\text { TT vs GG }\end{array}$ & $\begin{array}{l}0.428 \\
0.545 \\
0.617\end{array}$ \\
\hline
\end{tabular}




\section{Kidney Blood Pressure Research}

Table 3. CASR rs1801725 genotype and allele frequencies in HD patients with serum PTH $>437 \mathrm{pg} / \mathrm{mL}$ and $\leq 437 \mathrm{pg} / \mathrm{mL}$. $\mathrm{P}$ values significant after a Bonferroni correction $(\mathrm{P}<0.017)$ are indicated using bold font. Abbreviations: HD, hemodialysis; HWE, Hardy-Weinberg equilibrium; MAF, minor allele frequency; PTH, parathyroid hormone

\begin{tabular}{|c|c|c|c|c|}
\hline CASR rs1801725 & $\begin{array}{l}\text { HD patients with serum PTH }>437 \mathrm{pg} / \mathrm{mL} \\
\qquad \begin{array}{c}\text { (n, frequency) } \\
n=503\end{array}\end{array}$ & $\begin{array}{c}\text { HD patients serum PTH } \\
\leq 437 \mathrm{pg} / \mathrm{mL} \\
\text { (n, frequency) } \\
\mathrm{n}=712\end{array}$ & Odds ratio $(95 \% \mathrm{CI})$ & $P$ value \\
\hline \multicolumn{5}{|c|}{$P_{\text {trend }}=0.002, P_{\text {genotype }}=0.007$} \\
\hline GG & $323(64.2)$ & $517(72.6)$ & Reference & \\
\hline GT & $166(33.0)$ & $181(25.4)$ & $1.468(1.141-1.889)$ & 0.003 \\
\hline TT & $14(2.8)$ & $14(2.0)$ & $1.601(0.753-3.401)$ & 0.217 \\
\hline $\mathrm{TT}+\mathrm{GT}$ vs GG & $180(35.8)$ & $195(27.4)$ & $1.477(1.156-1.889)$ & 0.002 \\
\hline TT vs $\mathrm{GT}+\mathrm{GG}$ & $14(2.8)$ & $14(2.0)$ & $1.427(0.674-3.021)$ & 0.350 \\
\hline MAF & $(0.19)$ & $(0.15)$ & $1.389(1.121-1.721)$ & 0.003 \\
\hline $\mathrm{P}$ for HWE & 0.178 & 0.689 & & \\
\hline
\end{tabular}

Table 4. Associations of $C A S R$ rs 1801725 polymorphic variants with the lowest calcium concentrations and the highest phosphorus levels among the respective last four collected values of hemodialysis patients ( $\mathrm{n}=$ 1215). ${ }^{a}$ Mann Whitney test. Results are presented as median and range (minimum-maximum). Conversion factors to SI units are as follows: for calcium $-1 \mathrm{mg} / \mathrm{dL}=0.25 \mathrm{mmol} / \mathrm{L}$, for phosphorus $-1 \mathrm{mg} / \mathrm{dL}=0.323$ $\mathrm{mmol} / \mathrm{L}$

\begin{tabular}{|c|c|c|c|c|c|}
\hline Parameter & GG & GT & TT & $\begin{array}{c}\text { Mode } \\
\text { of inheritance }\end{array}$ & P value \\
\hline Minimum total calcium, mg/dL $\mathrm{n}=1165$ & $\begin{array}{c}8.32 \\
(4.64-11.5)\end{array}$ & $\begin{array}{c}8.4 \\
(6.1-10.74)\end{array}$ & $\begin{array}{c}8.4 \\
(6.5-10.2)\end{array}$ & $\begin{array}{c}\text { TT+ GT vs GG } \\
\text { TT vs. GT+GG } \\
\text { TT vs GG }\end{array}$ & $\begin{array}{l}0.367^{a} \\
0.977^{a} \\
0.905^{a}\end{array}$ \\
\hline Maximum phosphorus, mg/dL $n=1211$ & $\begin{array}{c}5.8 \\
(2.17-13.17)\end{array}$ & $\begin{array}{c}6.0 \\
(2.1-13.0)\end{array}$ & $\begin{array}{c}5.7 \\
(3.65-9.3)\end{array}$ & $\begin{array}{c}\text { TT+ GT vs GG } \\
\text { TT vs GT+GG } \\
\text { TT vs GG }\end{array}$ & $\begin{array}{l}0.085^{\mathrm{a}} \\
0.863^{\mathrm{a}} \\
0.721^{\mathrm{a}}\end{array}$ \\
\hline
\end{tabular}

\section{CASR SNP as an independent determinant of serum PTH level}

Gender, age at RRT onset, RRT duration, diabetic nephropathy, serum Ca and P concentrations, serum ALP activity, 25-hydroxyvitamin D, and the variant allele of CASR rs 1801725 were chosen as possible associates of serum PTH concentration. CASR rs1801725 $\mathrm{T}$ allele was a significant positive predictor of serum PTH concentration together with RRT duration, $\mathrm{Ca}$ and P concentrations, and total ALP activity. Increasing age and diabetic nephropathy showed a decreasing effect on PTH levels (adjusted P $=0.009$, Table 5).

\section{Response to treatment with cinacalcet}

Patients stratified by CASR rs1801725 genotypes and analyzed in models of inheritance did not differ significantly in serum PTH at the therapy onset and maximum doses of cinacalcet (Table 6). There was no significant difference in prevalence of responders to cinacalcet concerning the CASR rs1801725 polymorphism (Table 7).

\section{CASR haplotypes}

CASR rs7652589_rs1801725 AT haplotype (composed of two variant alleles) was significantly more prevalent $\left(\mathrm{P}_{\text {corr }}=0.021\right)$ in HD patients with serum PTH over $437 \mathrm{pg} /$ $\mathrm{mL}$ and associated with about 1.7 -fold higher frequency of plasma PTH values over $437 \mathrm{pg} /$ $\mathrm{mL}$ than reference haplotype composed of two major alleles. CASR rs7652589_rs1801725 AG haplotype was 1.5-fold more frequent in nephrolithiasis-related ESRD than rs7652589. rs1801725 GG haplotype ( $\mathrm{P}=0.004)$ (Table 8).

CASR haplotypes were not associated with response to treatment with cinacalcet

(Table 9). 


\section{Kidney Blood Pressure Research}

Table 5. Associates of serum PTH concentration in HD patients $(n=1215)$. a $-\beta$ coefficient and SE values can be interpreted as follows: for a unitary change in an analyzed parameter, serum PTH concentration would change by $\beta \pm \mathrm{SE}, \mathrm{pg} / \mathrm{mL}$. $\mathrm{b}$ - adjustment for variables significant in univariate analyses. $\mathrm{c}-\mathrm{n}=245$. Abbreviations: ALP - alkaline phosphatase, CASR - calcium-sensing receptor gene, HD - hemodialysis, PTH - parathyroid hormone, RRT - renal replacement therapy

\begin{tabular}{lccc}
\hline Parameter & $\beta \pm \mathrm{SE}^{\mathrm{a}}$ & $\mathrm{P}$ value & Adjusted P value $^{\mathrm{b}}$ \\
\hline Male gender & $-38.6 \pm 29.5$ & 0.191 & 0.595 \\
Age at RRT onset (per 10 years) & $-88.5 \pm 9.1$ & $2.0 \mathrm{E}-21$ & 0.0002 \\
RRT duration (per 1 year) & $36.6 \pm 3.4$ & $1.5 \mathrm{E}-26$ & $2.3 \mathrm{E}-13$ \\
Diabetic nephropathy & $-134.4 \pm 31.6$ & $2.3 \mathrm{E}-5$ & 0.0001 \\
Ca (per 1 mg/dL) & $61.5 \pm 18.8$ & 0.001 & 0.005 \\
P (per 1 mg/dL) & $77.3 \pm 9.8$ & $6.2 \mathrm{E}-15$ & $\approx 0$ \\
ALP (per 1 U/L) & $1.86 \pm 0.11$ & $\approx 0$ & $\approx 0$ \\
25-hydroxyvitamin D (per 1 ng/mL)c & $-4.57 \pm 5.34$ & 0.393 & 0.935 \\
CASR rs1801725 T allele & $41.3 \pm 15.8$ & 0.009 & 0.009 \\
\hline
\end{tabular}

Table 6. Effects of treatment with cinacalcet concerning CASR rs1801725 polymorphic variants in hemodialysis patients. Results are presented as median and range (minimum-maximum) or number (percentage). a - Mann-Whitney U test for quantitative variables or Chi-squared test for qualitative variables. b - Fisher's test

\begin{tabular}{|c|c|c|c|c|c|}
\hline \multirow[b]{2}{*}{ Parameter } & \multicolumn{3}{|c|}{ CASR rs1801725 } & \multirow{2}{*}{$\begin{array}{c}\text { Model of } \\
\text { inheritance }\end{array}$} & \multirow[b]{2}{*}{ P value } \\
\hline & $\begin{array}{c}\mathrm{GG} \\
\mathrm{n}=158\end{array}$ & $\begin{array}{c}\mathrm{GT} \\
\mathrm{n}=82\end{array}$ & $\begin{array}{c}\mathrm{TT} \\
\mathrm{n}=5\end{array}$ & & \\
\hline Maximum dose of cinacalcet, $\mathrm{mg} /$ day & $60(30-150)$ & $60(30-180)$ & $60(30-90)$ & $\begin{array}{c}\text { TT + GT vs GG } \\
\text { TT vs GT + GG } \\
\text { TT vs GG }\end{array}$ & $\begin{array}{l}0.576 \\
0.826 \\
0.781\end{array}$ \\
\hline $\begin{array}{l}\text { Parathyroid hormone at the therapy } \\
\text { onset, } \mathrm{pg} / \mathrm{mL}\end{array}$ & $(505-3280)$ & $(510.4-2991.5)$ & $\begin{array}{c}1146.5 \\
(550-1559)\end{array}$ & $\begin{array}{l}\text { TT + GT vs GG } \\
\text { TT vs GT + GG } \\
\text { TT vs GG }\end{array}$ & $\begin{array}{l}0.452 \\
0.836 \\
0.725\end{array}$ \\
\hline $\begin{array}{l}\text { Parathyroid hormone at } 1-2 \text { months } \\
\text { after the application of the maximum } \\
\text { dose, } \mathrm{pg} / \mathrm{mL}\end{array}$ & $\begin{array}{c}439.3 \\
(27.1-3281)\end{array}$ & $\begin{array}{c}395.6 \\
(16.2-2495.2)\end{array}$ & $\begin{array}{c}318 \\
(157-1059)\end{array}$ & $\begin{array}{l}\text { TT + GT vs GG } \\
\text { TT vs GT + GG } \\
\text { TT vs GG }\end{array}$ & $\begin{array}{l}0.727 \\
0.823 \\
0.806\end{array}$ \\
\hline $\begin{array}{l}\text { Percentual decrease of the initial } \\
\text { parathyroid hormone concentration }\end{array}$ & $\begin{array}{c}53.7 \\
(-122.2-96.7)\end{array}$ & $\begin{array}{c}55.2 \\
(-113.4-97.8)\end{array}$ & $56(7.6-89.9)$ & $\begin{array}{c}\text { TT + GT vs GG } \\
\text { TT vs GT + GG } \\
\text { TT vs GG }\end{array}$ & $\begin{array}{l}0.388 \\
0.947 \\
0.866\end{array}$ \\
\hline Response to cinacalcet, $n, \%$ of cases & $115(72.8)$ & $63(76.8)$ & $4(80.0)$ & $\begin{array}{l}\text { TT + GT vs GG } \\
\text { TT vs GT + GG } \\
\text { TT vs GG }\end{array}$ & $\begin{array}{l}0.469 \\
1.000^{\mathrm{b}} \\
1.000^{\mathrm{b}}\end{array}$ \\
\hline
\end{tabular}

Table 7. CASR rs1801725 genotype and allele frequencies in HD patients stratified by responsiveness to treatment with cinacalcet. a - Pearson's Chi-squared test, b - Cochran-Armitage Trend Test, c - Fisher's test. Abbreviations: HD, hemodialysis; HWE, Hardy-Weinberg equilibrium; MAF, minor (variant) allele frequency

\begin{tabular}{|c|c|c|c|c|}
\hline CASR rs1801725 & $\begin{array}{l}\text { Non-responders to cinacalcet } \\
\text { (n, frequency) } \\
n=63\end{array}$ & $\begin{array}{c}\text { Responders to cinacalcet } \\
\text { (n, frequency) } \\
n=182\end{array}$ & Odds ratio $(95 \% \mathrm{CI})$ & P value ${ }^{a}$ \\
\hline \multicolumn{5}{|c|}{$\mathrm{P}_{\text {trend }}=0.459 \mathrm{~b}, \mathrm{P}_{\text {genotype }}=0.817 \mathrm{c}$} \\
\hline GG & $43(68.3)$ & $115(63.2)$ & Reference & \\
\hline GT & $19(30.2)$ & $63(34.6)$ & $0.807(0.433-1.501)$ & 0.497 \\
\hline TT & $1(1.6)$ & $4(2.2)$ & $0.669(0.073-6.151)$ & $1.000^{\mathrm{b}}$ \\
\hline $\mathrm{TT}+\mathrm{GT}$ vs GG & $20(31.7)$ & $67(36.8)$ & $0.798(0.434-1.469)$ & 0.469 \\
\hline TT vs GT+GG & $1(1.6)$ & $4(2.2)$ & $0.718(0.079-6.545)$ & $1.000^{\mathrm{b}}$ \\
\hline MAF & $(0.17)$ & $(0.20)$ & $0.825(0.483-1.410)$ & 0.482 \\
\hline$P$ for HWE & 0.496 & 0.167 & & \\
\hline
\end{tabular}




\section{Kidney \\ Blood Pressure Research}

Table 8. CASR rs7652589_rs1801725 haplotypes and prevalence of plasma PTH levels over $437 \mathrm{pg} / \mathrm{mL}$ and nephrolithiasis-related ESRD. ${ }^{a} \mathrm{P}$ value calculated using permutation test and a total of 1,000 permutations, ${ }^{\mathrm{b}}$ The most common haplotype was used as the reference, ${ }^{\mathrm{c}}$ All other haplotypes pooled together were used as the reference. Bold font indicates $\mathrm{P}$ values significant after correction; OR (95\%CI) were considered significant only if their $\mathrm{P}$ values were $<0.05$ and simultaneously associated with significant $\mathrm{P}$ values calculated using permutation test and a total of 1,000 permutations

\begin{tabular}{|c|c|c|c|c|c|c|c|}
\hline Haplotype & Freq. & Case,Control Frequencies & Chi Square & P Value & $\mathrm{P}_{\text {corr Value }}$ & OR (95\%Cl); p value & OR $(95 \% \mathrm{CI})$; p valuec \\
\hline \multicolumn{8}{|c|}{ Plasma parathyroid hormone levels over $437 \mathrm{pg} / \mathrm{mL}$} \\
\hline GG & 0.525 & $0.486,0.553$ & 10.564 & 0.0012 & 0.0020 & reference & $0.766(0.651-0.900) ; 0.0012$ \\
\hline AG & 0.309 & $0.321,0.300$ & 1.191 & 0.2750 & 0.5510 & $1.215(1.011-1.459) ; 0.0376$ & $1.101(0.925-1.310) ; 0.2811$ \\
\hline GT & 0.092 & $0.103,0.085$ & 2.254 & 0.1333 & 0.2910 & $1.370(1.029-1.824) ; 0.0305$ & $1.228(0.932-1.619) ; 0.1439$ \\
\hline AT & 0.074 & $0.090,0.062$ & 6.868 & 0.0088 & 0.0210 & $1.664(1.216-2.278) ; 0.0014$ & $1.510(1.113-2.048) ; 0.0077$ \\
\hline \multicolumn{8}{|c|}{ Nephrolithiasis-related ESRD } \\
\hline GG & 0.525 & $0.455,0.531$ & 4.269 & 0.0388 & 0.0730 & reference & $0.735(0.549-0.984) ; 0.0380$ \\
\hline AG & 0.309 & $0.399,0.301$ & 8.115 & 0.0044 & 0.0070 & $1.549(1.129-2.126) ; 0.0064$ & 1.541 (1.143 - 2.077); 0.0043 \\
\hline GT & 0.092 & $0.075,0.094$ & 0.726 & 0.3942 & 0.7400 & $0.946(0.537-1.666) ; 0.8469$ & $0.793(0.460-1.369) ; 0.4045$ \\
\hline AT & 0.074 & $0.071,0.074$ & 0.021 & 0.8844 & 0.9980 & $1.118(0.622-2.010) ; 0.7088$ & $0.953(0.541-1.679) ; 0.8681$ \\
\hline
\end{tabular}

Table 9. Haplotypes of CASR rs7652589_rs1801725 and responsiveness to treatment with cinacalcet in HD patients. ${ }^{a}$ p-value calculated using permutation test and a total of 1,000 permutations

\begin{tabular}{|c|c|c|c|c|c|c|}
\hline SNPS & Haplotype & Freq. & Case,Control Frequencies & Chi Square & P Value & $P_{\text {corr Value }}$ Va \\
\hline \multirow{4}{*}{ rs7652589_rs1801725 } & GG & 0.503 & $0.517,0.460$ & 1.228 & 0.2677 & 0.5690 \\
\hline & AG & 0.310 & $0.287,0.373$ & 3.214 & 0.0730 & 0.1530 \\
\hline & GT & 0.110 & $0.120,0.080$ & 1.567 & 0.2107 & 0.4590 \\
\hline & AT & 0.078 & $0.075,0.087$ & 0.187 & 0.6651 & 0.9510 \\
\hline
\end{tabular}

Epistatic interactions between CASR rs1801725 and vitamin D signaling pathway genes

Epistatic interactions between the CASR rs1801725 SNP and vitamin D signaling pathway gene SNPs were examined in HD patients with vs. without serum PTH concentration over $437 \mathrm{pg} / \mathrm{mL}$, responsiveness vs. unresponsiveness to treatment with cinacalcet, and nephrolithiasis-related ESRD vs. other causes of ESRD. There were no significant interactions between CASR rs1801725 and vitamin D signaling pathway gene SNPs concerning tested phenotypes (Tables 10-12).

Association of the CASR rs1801725 SNP with clinical outcomes of HD patients

A total of $706 \mathrm{HD}$ patients died, and 161 underwent RTx between January 2nd, 2009 and January 25th, 2018.

Using the Kaplan-Meier curve estimate in the entire cohort of HD patients, CASR rs1801725 genotypes did not show an effect on all-cause (log-rank test $\mathrm{P}=0.395$ ), cardiovascular (log-rank test $\mathrm{P}=0.594$ ), neoplasm-related (log-rank test $\mathrm{P}=0.543$ ), and infection-related (log-rank test $\mathrm{P}=0.083$ ) mortality. However, Cox proportional hazard model showed that homozygotes of the variant allele of CASR rs1801725 SNP had higher infection-related mortality compared with heterozygotes and homozygotes of the major allele (Figures 1-4). Homozygosity of the CASR rs1801725 variant allele was associated with infection-related mortality in the recessive $(P=0.003$, Fig. 3$)$ and additive $(P=0.005$, Fig. 4) models of inheritance. If gender, age at RRT onset, diabetic nephropathy, and CAD/MI in the course of RRT were included in the model, only age and CASR rs1801725 SNP were associated with infection-related mortality (Table 13). 


\section{Kidney Blood Pressure Research}

Table 10. Epistatic interactions between the CASR rs1801725 SNP and vitamin D signaling pathway gene SNPs in HD patients with vs. without serum PTH concentration over $437 \mathrm{pg} / \mathrm{mL}$. a - Significance of accuracy, empirical $\mathrm{P}$ value based on 1,000 permutations

\begin{tabular}{lrr}
\hline Genes and rs numbers & Testing Balanced Accuracy & Cross Validation Consistency $^{\mathrm{p} \text { value }}$ \\
\hline$G C_{-}$rs7041, CASR_rs1801725 & 0.5125 & $6 / 10$ \\
$V D R_{-}$rs2228570, RXRA_rs10776909, GC_rs7041 & 0.5330 & $5 / 10$ \\
$V D R_{-}$rs2228570, RXRA_rs10776909, GC_rs7041, CASR_rs1801725 & 0.4747 & 0.5410 \\
\hline
\end{tabular}

Table 11. Epistatic interactions between the CASR rs1801725 SNP and vitamin D signaling pathway gene SNPs in HD patients with responsiveness vs. unresponsiveness to treatment with cinacalcet. a - Significance of accuracy, empirical $P$ value based on 1,000 permutations

\begin{tabular}{|c|c|c|c|}
\hline Genes and rs numbers & Testing Balanced Accuracy & Cross Validation Consistency & Pvalue ${ }^{a}$ \\
\hline CASR_rs7652589, RXRA_rs10776909 & 0.4860 & $4 / 10$ & 0.9160 \\
\hline$V D R_{-} r \mathrm{rs} 2228570, V D R_{-} r \mathrm{r} 1544410, G C_{-} \mathrm{rs} 2298849$ & 0.5290 & $5 / 10$ & 0.6700 \\
\hline CASR_rs7652589, VDR_rs2228570, VDR_rs1544410, GC_rs7041 & 0.5275 & $6 / 10$ & 0.6830 \\
\hline
\end{tabular}

Table 12. Epistatic interactions between the CASR rs1801725 SNP and vitamin D signaling pathway gene SNPs in HD patients with nephrolithiasis-related ESRD vs. other causes of ESRD. a - Significance of accuracy, empirical $P$ value based on 1,000 permutations

\begin{tabular}{lcr}
\hline Genes and rs numbers & Testing Balanced Accuracy & Cross Validation Consistency $^{\text {P value }}$ \\
\hline VDR_rs1544410, GC_rs2298849 & 0.5010 & $5 / 10$ \\
VDR_rs1544410, GC_rs2298849, CASR_rs1801725 & 0.4767 & $4 / 10$ \\
VDR_rs2228570, VDR_rs1544410, RXRA_rs10881578, GC_rs7041 & 0.4464 & 0.9660 \\
\hline
\end{tabular}

Fig. 1. The Kaplan-Meier survival analysis of infectionrelated mortality in the studied he modialysis $\mathrm{p}$ a $\mathrm{t}$ i e $\mathrm{n} \mathrm{t} \mathrm{s}$ stratified by CASR rs7652589 genotypes. logrank test $\mathrm{P}=$ 0.083; TT vs. GT: HR 7.95, 95\%Cl $2.15-29.37, \mathrm{P}=$ 0.003; TT vs. GG: HR 5.89, 95\%Cl $1.69-20.55, \mathrm{P}=$ 0.040 .

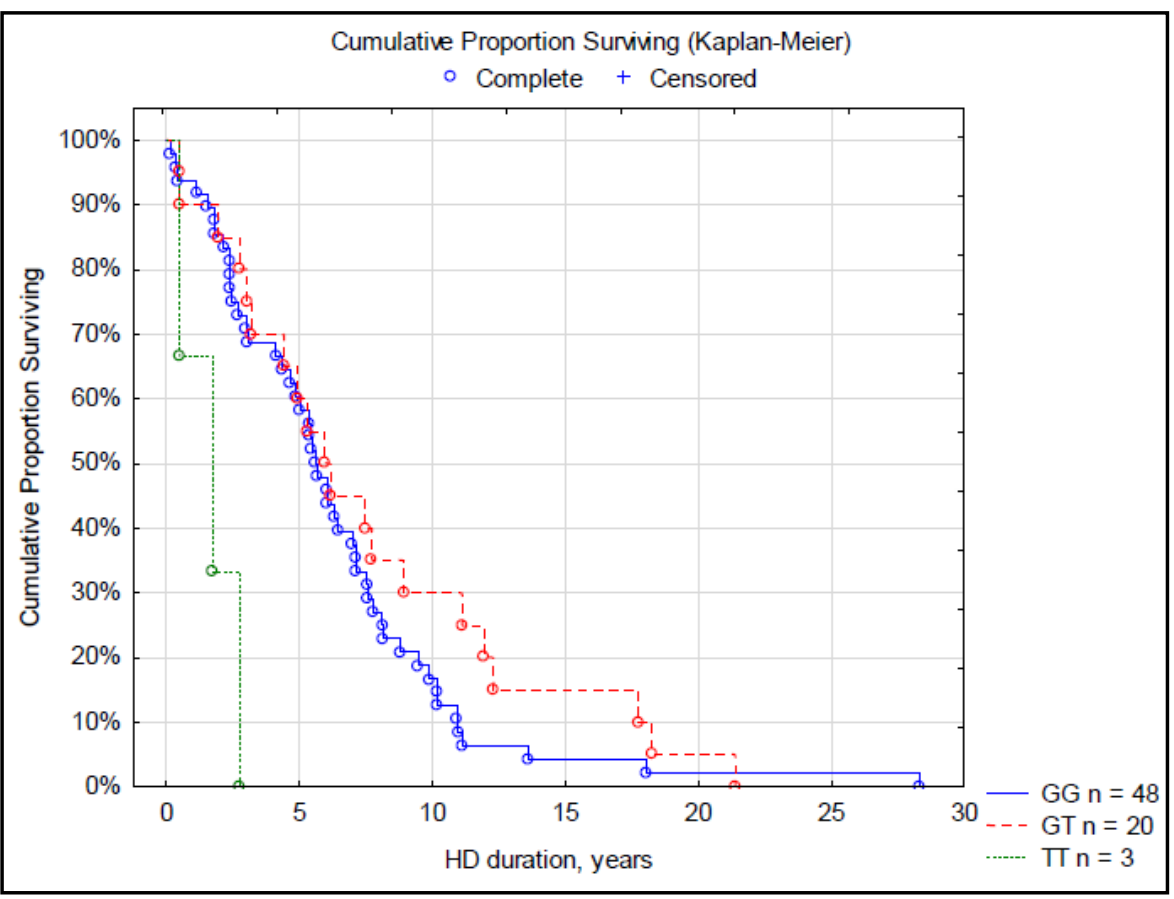




\section{Kidney Blood Pressure Research}

Fig. 2. The Kaplan-Meier survival analysis of infectionrelated mortality in the studied hemodialysis $\mathrm{p}$ a $\mathrm{t} i \mathrm{e} \mathrm{nt}$ stratified by CASR rs1801725 genotypes in the dominant model of inheritance. log-rank test $\mathrm{P}$ $=0.508$; Hazard ratio (HR) for death in TT + GT genotype group vs. GG genotype group: HR 0.85, $95 \% \mathrm{Cl} \quad 0.51 \quad-$ $1.41, \mathrm{P}=0.521$.

Fig. 3. The Kaplan-Meier survival analysis of infectionrelated mortality in the studied hemodialys is $\mathrm{p}$ a $\mathrm{t}$ i e $\mathrm{nt} \mathrm{s}$ stratified by CASR rs1801725 genotypes in the recessive model of inheritance. log-rank test $\mathrm{P}=0.137$; Hazard ratio (HR) for death in TT genotype group vs. GG+GT genotype group: $\mathrm{HR} 6.39,95 \% \mathrm{Cl}$ $1.85-22.14, \mathrm{P}=$ 0.003 .
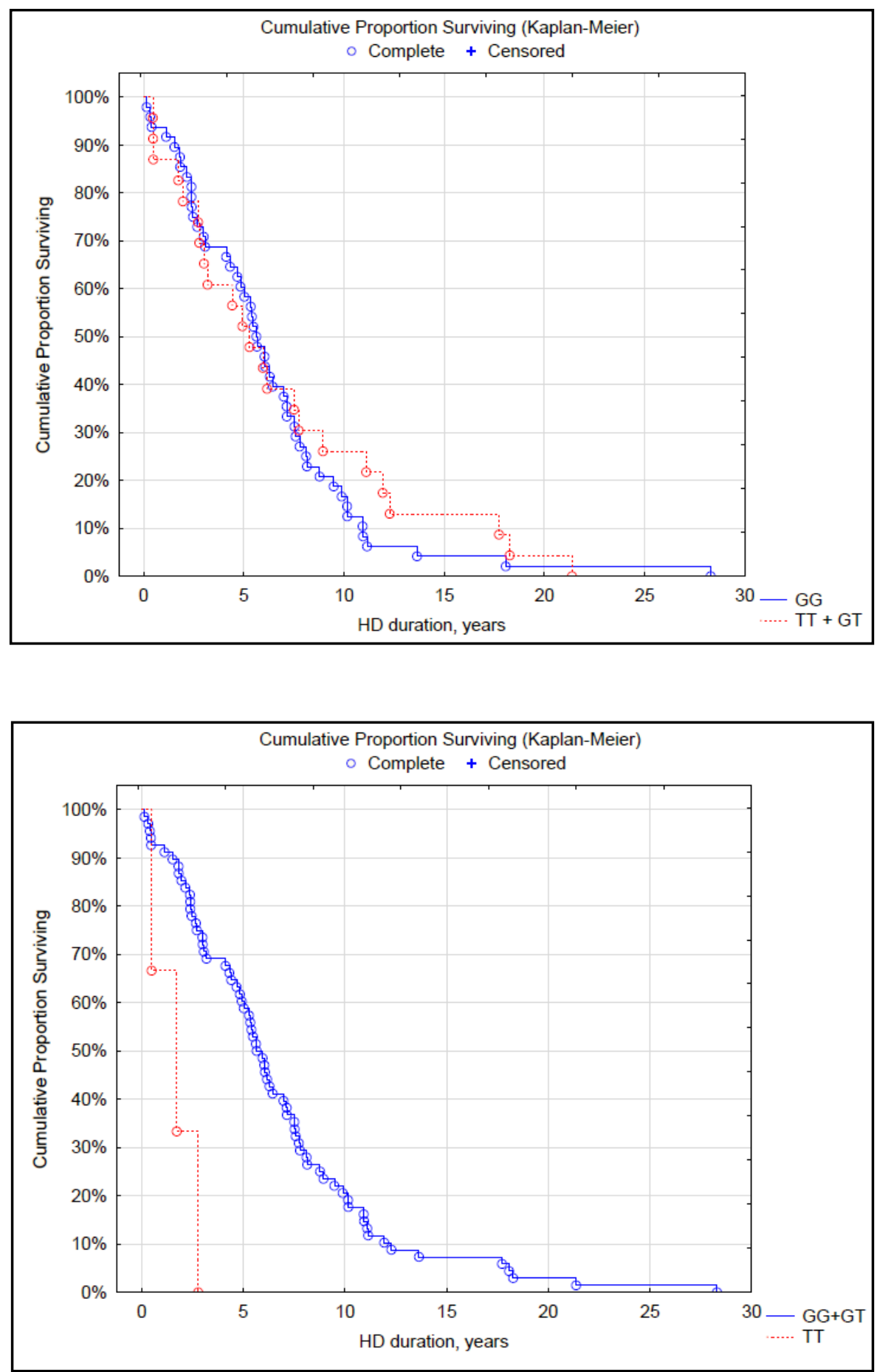


\section{Kidney Blood Pressure Research}

Fig. 4. The Kaplan-M e i e r survival analysis of infectionrelated mortality in the studied he modialys is $\mathrm{p}$ a $\mathrm{t} \mathrm{i}$ e $\mathrm{n} \mathrm{t} \mathrm{s}$ stratified by CASR rs1801725 genotypes in the additive model of inheritance. logrank test $\mathrm{P}=0.138$; Hazard ratio (HR) for death in TT genotype group vs. GG genotype group: HR 6.09, $95 \% \mathrm{Cl} \quad 1.71$ $21.74, \mathrm{P}=0.005$.

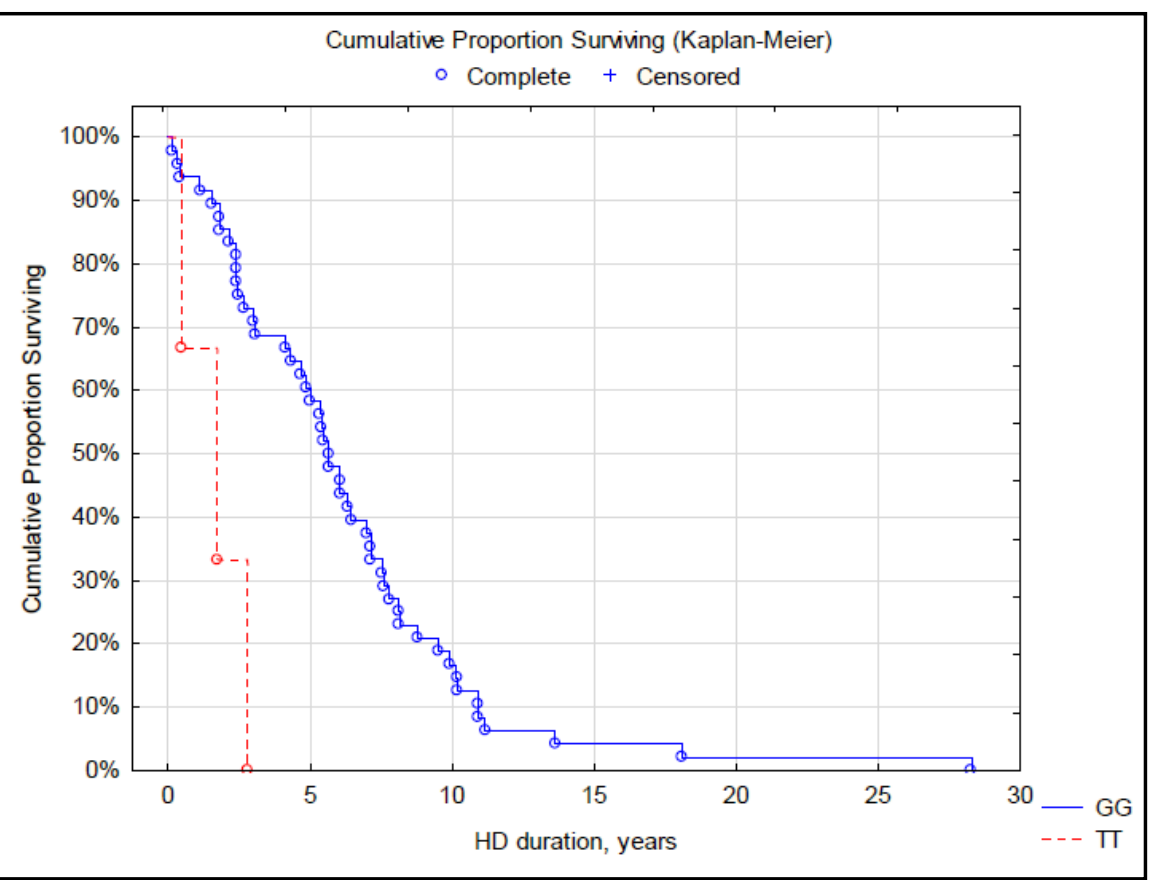

Table 13. Results of multivariate analyses using Cox proportional hazard model for prediction of variables explaining infection-related mortality in HD patients. a - Hazard ratio for this variable is calculated for an increase of age at RRT onset by one year. Abbreviations: CAD - coronary artery disease, CASR - calciumsensing receptor gene, HD - hemodialysis, MI - myocardial infarction, RRT - renal replacement therapy

\begin{tabular}{lcc}
\hline Parameters & $\begin{array}{c}\text { Gender, age at RRT onset, diabetic nephropathy, CAD, and CASR rs1801725 } \\
\text { polymorphic variants }\end{array}$ & $\begin{array}{c}\text { Gender, age at RRT onset, diabetic nephropathy, MI, and CASR rs1801725 } \\
\text { polymorphic variants }\end{array}$ \\
\hline Significant predictors & $\mathrm{HR}, 95 \%$ CI, P value & $\mathrm{HR}, 95 \%$ CI, P value \\
Age at RRT onseta & $1.03,1.00-1.05,0.047$ & $1.03,1.01-1.05,0.011$ \\
CASR rs1801725 GG vs. TT & $0.16,0.04-0.58,0.028$ & $0.16,0.04-0.60,0.026$ \\
CASR rs1801725 GT vs. TT & $0.13,0.03-0.51,0.008$ & $0.14,0.03-0.55,0.012$ \\
Age at RRT onseta & $1.02,1.01-1.05,0.012$ & $1.03,1.01-1.05,0.004$ \\
CASR rs1801725 GG vs. GT+TT & $\mathrm{NS}$ & $\mathrm{NS}$ \\
(dominant model of & & $1.03,1.01-1.05,0.006$ \\
inheritance) & $1.03,1.01-1.05,0.005$ & $0.16,0.04-0.59,0.006$ \\
Age at RRT onseta & $0.16,0.04-0.56,0.005$ & $0.12,0.03-0.48,0.003$ \\
CASR rs1801725 GG + GT vs. TT & & \\
(recessive model of & & \\
inheritance) & $0.12,0.03-0.46,0.002$ & \\
CASR rs1801725 GG vs. TT & & \\
(additive model of inheritance) & &
\end{tabular}

\section{Discussion}

In this study, we have shown that HD patients of European ancestry, who harbor the variant allele of CASR rs1801725, develop higher PTH levels than homozygotes of the major allele; therefore, they are prone to more severe sHPT. In this aspect, our study confirms data obtained by Moe et al. [7]. The variant allele of rs1801725 was an independent predictor of serum PTH levels among variables already associated with PTH concentrations in HD patients [1, 28-30]. Our previous study [6] demonstrated that the variant homozygosity of CASR rs7652589 was associated with PTH levels over $500 \mathrm{pg} / \mathrm{mL}$. The present data recognize CASR rs7652589_rs1801725 AT haplotype (composed of two variant alleles) as associated with more severe sHPT, and indicates that both CASR SNPs act in cooperation concerning serum PTH.

Calcimimetics increase CaSR expression [31] and are used to reduce PTH secretion by parathyroid cells in sHPT patients [20]. A decrease in serum PTH concentration in response 


\section{Kidney \\ Blood Pressure Research}

Kidney Blood Press Res 2018;43:719-734

\begin{tabular}{l|l}
\hline DOI: $10.1159 / 000489747$ & (C) 2018 The Author(s). Published by S. Karger AG, Basel
\end{tabular}

Published online: 16 May, 2018

www.karger.com/kbr

731

to administration of cinacalcet varies between HD patients, but there is no definite evidence showing that CASR SNPs have a direct impact on PTH-lowering response to cinacalcet, although several CASR SNPs were investigated for association [6-9]. In HD group receiving cinacalcet, we could not show any association between a decrease in serum PTH in response to cinacalcet and CASR rs1801725 SNP or CASR rs7652589_rs1801725 haplotypes.

In non-uremic subjects, CASR rs1801725 SNP was found as attributed to the development of calcium nephrolithiasis [32, 33] and simultaneously higher serum calcium levels [33] or not associated with calcium kidney stone formation [17, 34, 35]. In HD patients, CASR rs1801725 SNP did not associate solely either with nephrolithiasis-related ESRD or serum calcium concentration. Therefore, higher frequency of CASR rs7652589_rs1801725 AG haplotype in patients showing nephrolithiasis-related ESRD seems to be dependent on the rs7652589 A allele.

Although the variant allele of CASR rs1801725 appeared as associated with more advanced SHPT, it was not attributed in this retrospective study to CAD, MI, and all-cause or cardiovascular mortality of HD patients of European ancestry. In the 7-year prospective study, CASR rs7652589 was not associated with all-cause and cardiovascular mortality in HD subjects [26]. CASR polymorphic variants also had no impact on the occurrence of cardiovascular events and all-cause mortality in renal transplant recipients [36]. Similarly, bone fractures were not attributed to CASR rs1801725 [7]. Serum PTH concentration $\geq 600$ $\mathrm{mg} / \mathrm{mL}$ determined mortality of HD patients [30, 37]. The variant allele in CASR rs1801725 was associated with serum PTH concentrations over $437 \mathrm{pg} / \mathrm{mL}$. Therefore, PTH levels associated with this variant allele could be too low to separate HD survivors and nonsurvivors concerning serum PTH.

Homozygosity of the variant allele in CASR rs1801725 contributed to higher infectionrelated mortality of HD patients. Involvement of CaSR or its activators in infectious diseases was suggested in several studies [38, 39]. Human peripheral blood monocytes possess both CaR protein and mRNA [40]. CASR rs7652589 SNP interacted with CCL2 rs1024611 SNP concerning nephrolithiasis-related ESRD which was complicated with near $39 \%$ of cases with chronic infective tubulointerstitial nephritis [6]. In the 7-year prospective study, CASR rs7652589 yielded a borderline significance concerning infection-related mortality in the dominant model of inheritance [26]. However, how CASR rs1801725 SNP influences outcomes of infectious diseases remains unknown.

\section{Limitations of the study}

Associations of CASR rs1801725 with selected phenotypes were analyzed under conditions of conventional treatment targeting sHPT. Ca-containing drugs were administered the most commonly, serving as phosphate binders and Ca supplements. These drugs were usually started still before HD therapy, and their discontinuation was not possible due to ethical reasons. Although about a half of HD subjects is noncompliant with medication taking [41], Ca-containing drugs and vitamin D supplements could abolish differences in serum Ca and $P$ resulting from genetic variability.

\section{Acknowledgements}

This study was funded by the Poznan University of Medical Sciences, Poznań, Poland, grant numbers 502-01-02225363-03679 and 502-01-01124182-07474.

\section{Disclosure Statement}

The authors declare no competing financial interests. 


\section{Kidney \\ Blood Pressure Research}

\section{References}

1 Li J, Molnar MZ, Zaritsky JJ, Sim JJ, Streja E, Kovesdy CP, Salusky I, Kalantar-Zadeh K: Correlates of parathyroid hormone concentration in hemodialysis patients. Nephrol Dial Transplant 2013;28:1516-1525.

$\checkmark 2$ de Francisco ALM, Fresnedo GF, Rodrigo E, Piñera C, Amado JA, Arias M: Parathyroidectomy in dialysis patients. Kidney Int Suppl 2002:161-166.

3 U.S. Renal Data System, USRDS 2013 Annual Data Report: Atlas of Chronic Kidney Disease and EndStage Renal Disease in the United States, National Institutes of Health, National Institute of Diabetes and Digestive and Kidney Diseases, Bethesda, MD, 2013.

-4 Yano S, Sugimoto T, Kanzawa M, Tsukamoto T, Hattori T, Hattori S, Chihara K: Association of polymorphic alleles of the calcium-sensing receptor gene with parathyroid hormone secretion in hemodialysis patients. Nephron 2000;85:317-323.

-5 Yokoyama K, Shigematsu T, Tsukada T, Hara S, Yamada A, Kawaguchi Y, Hosoya T: Calcium-sensing receptor gene polymorphism affects the parathyroid response to moderate hypercalcemic suppression in patients with end-stage renal disease. Clin Nephrol 2002;57:131-135.

6 Grzegorzewska AE, Paciorkowski M, Mostowska A, Frycz B, Warchoł W, Stolarek I, Figlerowicz M, Jagodziński PP: Associations of the calcium-sensing receptor gene CASR rs7652589 SNP with nephrolithiasis and secondary hyperparathyroidism in haemodialysis patients. Sci Rep 2016;6:35188.

7 Moe SM, Wetherill L, Decker BS, Lai D, Abdalla S, Long J, Vatta M, Foroud TM, Chertow GM: Calcium-Sensing Receptor Genotype and Response to Cinacalcet in Patients Undergoing Hemodialysis. Clin J Am Soc Nephrol 2017;12:1128-1138.

8 Rothe HM, Shapiro WB, Sun WY, Chou SY: Calcium-sensing receptor gene polymorphism Arg990Gly and its possible effect on response to cinacalcet HCl. Pharmacogenet Genomics 2005;15:29-34.

9 Rothe HM, Shapiro WB, Sun WY, Matalon A: Calcium-sensing receptor gene polymorphism Arg990Gly influences the response to calcimimetic agents in end-stage kidney disease patients with secondary hyperparathyroidism. Personalized Med 2008;5:109-116.

10 Brown EM, Gamba G, Riccardi D, Lombardi M, Butters R, Kifor O, Sun A, Hediger MA, Lytton J, Hebert SC: Cloning and characterization of an extracellular Ca2+-sensing receptor from bovine parathyroid. Nature 1993:366:575-580.

$>11$ Cole DE, Peltekova VD, Rubin LA, Hawker GA, Vieth R, Hawker GA, Vieth R, Liew CC, Hwang DM, Evrovski J, Hendy GN: A986S polymorphism of the calcium-sensing receptor and circulating calcium concentrations. Lancet 1999;353:112-115.

12 Wang L, Widatalla SE, Whalen DS, Ochieng J, Sakwe AM: Association of calcium sensing receptor polymorphisms at rs1801725 with circulating calcium in breast cancer patients. BMC Cancer 201;17:511.

13 Miedlich S, Lamesch P, Mueller A, Paschke R: Frequency of the calcium-sensing receptor variant A986S in patients with primary hyperparathyroidism. Eur J Endocrinol 2001;145:421-427.

14 Harding B, Curley AJ, Hannan FM, Christie PT, Bowl MR, Turner JJ, Barber M, Gillham-Nasenya I, Hampson G, Spector TD, Thakker RV: Functional characterization of calcium sensing receptor polymorphisms and absence of association with indices of calcium homeostasis and bone mineral density. Clin Endocrinol (Oxf) 2006;65:598-605.

15 Vezzoli G, Terranegra A, Arcidiacono T, Biasion R, Coviello D, Syren ML, Paloschi V, Giannini S, Mignogna G, Rubinacci A, Ferraretto A, Cusi D, Bianchi G, Soldati L: R990G polymorphism of calcium-sensing receptor does produce a gain-of-function and predispose to primary hypercalciuria. Kidney Int 2007;71:1155-1162.

-16 Robinson-Cohen C, Lutsey PL, Kleber ME, Nielson CM, Mitchell BD, Bis JC, Eny KM, Portas L, Eriksson J, Lorentzon M, Koller DL, Milaneschi Y, Teumer A, Pilz S, Nethander M, Selvin E, Tang W, Weng LC, Wong HS, Lai D, et al.: Genetic Variants Associated with Circulating Parathyroid Hormone. J Am Soc Nephrol 2017:28:1553-1565.

17 Ding Q Fan B, Shi Y, Fan Z, Ding L, Li F, Tu W, Jin X, Qin C, Cao Q Yuan Q, Wang J, Ouyang J: Calcium-Sensing Receptor Genetic Polymorphisms and Risk of Developing Nephrolithiasis in a Chinese Population. Urol Int 2017;99:331-337. 


\section{Kidney \\ Blood Pressure Research}

18 Kapur K, Johnson T, Beckmann ND, Sehmi J, Tanaka T, Kutalik Z, Styrkarsdottir U, Zhang W, Marek D, Gudbjartsson DF, Milaneschi Y, Holm H, Diiorio A, Waterworth D, Li Y, Singleton AB, Bjornsdottir US, Sigurdsson G, Hernandez DG, Desilva R, et al.: Genome-wide meta-analysis for serum calcium identifies significantly associated SNPs near the calcium-sensing receptor (CASR) gene. PLoS Genet 2010;22:e1001035.

19 Marz W, Seelhorst U, Wellnitz B, Tiran B, Obermayer-Pietsch B, Renner W, Boehm BO, Ritz E, Hoffmann MM: Alanine to serine polymorphism at position 986 of the calcium-sensing receptor associated with coronary heart disease, myocardial infarction, all-cause, and cardiovascular mortality. J Clin Endocrinol Metab 2007;92:3263-2369.

20 Debelle F, Meeus G, Dratwa M, Maes B, Vanholder R, Albert A, Schutyser E, Jadoul M: Cinacalcet for managing secondary hyperparathyroidism in dialysis patients in clinical practice in Belgium: a 16-month observational study (ECHO-B). Acta Clin Belg 2013;68:275-281.

21 Bonarek H, Merville P, Bonarek M, Moreau K, Morel D, Aparicio M, Potaux L: Reduced parathyroid functional mass after successful kidney transplantation. Kidney Int 1999;56:642-649.

-22 Evenepoel P, Claes K, Kuypers D, Maes B, Bammens B, Vanrenterghem Y: Natural history of parathyroid function and calcium metabolism after kidney transplantation: a single-centre study. Nephrol Dial Transplant 2004;19:1281-1287.

23 US Renal Data System 2016 Annual Data Report: Epidemiology of Kidney Disease in the United States. Am J Kidney Dis DOI: 10.1053/j.ajkd.2016.12.004.

$\$ 24$ Kidney Disease: Improving Global Outcomes (KDIGO) CKD-MBD Work Group: KDIGO clinical practice guideline for the diagnosis, evaluation, prevention, and treatment of chronic kidney disease-mineral and bone disorder (CKD-MBD). Kidney Int Suppl 2009;S1-130.

25 Kidney Disease: Improving Global Outcomes (KDIGO) CKD-MBD Update Work Group: KDIGO 2017 Clinical Practice Guideline Update for the Diagnosis, Evaluation, Prevention, and Treatment of Chronic Kidney Disease-Mineral and Bone Disorder (CKD-MBD). Kidney Int Suppl DOI: 10.1016/j.kisu.2017.04.001.

-26 Grzegorzewska AE, Świderska MK, Mostowska A, Warchoł W, Jagodziński PP: Polymorphisms of Vitamin D Signaling Pathway Genes and Calcium-Sensing Receptor Gene in respect to Survival of Hemodialysis Patients: A Prospective Observational Study. Int J Endocrinol 2016;2016:2383216.

27 Hahn LW, Ritchie MD, Moore JH: Multifactor dimensionality reduction software for detecting gene-gene and gene-environment interactions. Bioinformatics 2003;19:376-382.

28 Mehrotra R, Supasyndh O, Berman N, Kaysen G, Hurst L, Leonardi M, Das D, Kopple JD: Age-related decline in serum parathyroid hormone in maintenance hemodialysis patients is independent of inflammation and dietary nutrient intake. J Ren Nutr 2004;14:134-142.

29 Murakami R, Murakami S, Tsushima R, Ueda C, Mikami K, Ebina T, Kumasaka R, Nakamura N, Okumura $\mathrm{K}$ : Glycaemic control and serum intact parathyroid hormone levels in diabetic patients on haemodialysis therapy. Nephrol Dial Transplant 2008;23:315-320.

-30 Soleymanian T, Nikzad N, Mahjoub A, Argani H, Saavaj S: Serum Levels of Intact Parathyroid Hormone, Calcium, and Phosphorus and Risk of Mortality in Hemodialysis Patients. Nephro-Urol Mon 2016; DOI: 10.5812/numonthly.42569.

-31 Hénaut L, Boudot C, Massy ZA, Lopez-Fernandez I, Dupont S, Mary A, Drüeke TB, Kamel S, Brazier M, Mentaverri R: Calcimimetics increase CaSR expression and reduce mineralization in vascular smooth muscle cells: mechanisms of action. Cardiovasc Res 2014;101:256-265.

-32 Shakhssalim N, Kazemi B, Basiri A, Houshmand M, Pakmanesh H, Golestan B, Eilanjegh AF, Kashi AH, Kilani M, Azadvari M: Association between calcium-sensing receptor gene polymorphisms and recurrent calcium kidney stone disease: a comprehensive gene analysis. Scand J Urol Nephrol 2010;4:406-412.

-33 Guha M, Bankura B, Ghosh S, Pattanayak AK, Ghosh S, Pal DK, Puri A, Kundu AK, Das M: Polymorphisms in CaSR and CLDN14 Genes Associated with Increased Risk of Kidney Stone Disease in Patients from the Eastern Part of India. PLoS One 2015;10:e0130790.

-34 Ferreira LG, Pereira AC, Heilberg IP: Vitamin D receptor and calcium-sensing receptor gene polymorphisms in hypercalciuric stone-forming patients. Nephron Clin Pract 2010;114:c135-144.

-35 Petrucci M, Scott P, Ouimet D, Trouvé ML, Proulx Y, Valiquette L, Guay G, Bonnardeaux A: Evaluation of the calcium-sensing receptor gene in idiopathic hypercalciuria and calcium nephrolithiasis. Kidney Int 2000;58:38-42. 


\section{Kidney Research}

Grzegorzewska et al.: Calcium-Sensing Receptor Gene in HD Patients

-36 Babinsky VN, Hannan FM, Youhanna SC, Maréchal C, Jadoul M, Devuyst O, Thakker RV: Association studies of calcium-sensing receptor (CaSR) polymorphisms with serum concentrations of glucose and phosphate, and vascular calcification in renal transplant recipients. PLoS One 2015;10:e0119459.

37 Block GA, Klassen PS, Lazarus JM, Ofsthun N, Lowrie EG, Chertow GM: Mineral metabolism, mortality, and morbidity in maintenance hemodialysis. J Am Soc Nephrol 2004;15:2208-2218.

-38 Cheng SX, Lightfoot YL, Yang T, Zadeh M, Tang L, Sahay B, Wang GP, Owen JL, Mohamadzadeh M: Epithelial CaSR deficiency alters intestinal integrity and promotes proinflammatory immune responses. FEBS Lett 2014;588:4158-4166.

39 Cheng SX: Calcium-sensing receptor: A new target for therapy of diarrhea. World J Gastroenterol 2016;22:2711-2724.

40 Yamaguchi T, Olozak I, Chattopadhyay N, Butters RR, Kifor O, Scadden DT, Brown EM: Expression of extracellular calcium ( $\mathrm{Ca} 2+0)$-sensing receptor in human peripheral blood monocytes. Biochem Biophys Res Commun 1998;246:501-506.

41 Bame SI, Petersen N, Wray NP: Variation in hemodialysis patient compliance according to demographic characteristics. Soc Sci Med 1993;37:1035-1043. 